\title{
Entanglement of Qutrits
}

\author{
A. A. Klyachko, B. Öztop*, and A. S. Shumovsky \\ Faculty of Science, Bilkent University, Bilkent, Ankara, 06800 Turkey \\ *e-mail: boztop@fen.bilkent.edu.tr \\ Received July 26, 2006
}

\begin{abstract}
For two types of qutrits specified by the dynamical symmetry SU(3) and SU(2), we consider the difference in entanglement caused by the lack of quantum observables in the latter case. In particular, we show that the $\mathrm{SU}(2)$ qutrits can have specific separable entanglement caused by quantum correlations of intrinsic degrees of freedom in a single party without interparty correlations.
\end{abstract}

PACS numbers: 03.67.Mn, 03.65.Ud, 03.67.-a

DOI: $10.1134 / \mathrm{S} 1054660 \mathrm{X} 07020302$

The main objective of this note is to accentuate the principle difference between the two possible realizations of qutrits with different dynamic symmetry properties.

Since the pioneering paper by late Professor David Klyshko and his coauthors [1], the problem of qutrits is widely discussed in the context of quantum ternary logic (see [2-6] and references therein). Qutrit is often defined as a quantum three-level or ternary system, that is, a system with states in the three-dimensional Hilbert space $\mathcal{H}_{3}$. We denote the orthonormal basis of $\mathscr{H}_{3}$ as follows:

$$
|+1\rangle,|0\rangle,|-1\rangle \text {. }
$$

In fact, the above definition of qutrits is incomplete. The point is that the general symmetry of the threelevel system is given by the group $G=\mathrm{SU}(3)$. This group is induced by the Lie algebra $\mathscr{L}=s u(3)$

$$
G=\exp (i \mathscr{L})
$$

whose orthogonal basis consists of eight independent Hermitian operators (Gell-Mann matrices) [7]

$$
\begin{array}{ll}
\lambda_{1}=\left(\begin{array}{lll}
0 & 1 & 0 \\
1 & 0 & 0 \\
0 & 0 & 0
\end{array}\right), & \lambda_{2}=\left(\begin{array}{ccc}
0 & -i & 0 \\
i & 0 & 0 \\
0 & 0 & 0
\end{array}\right), \\
\lambda_{3}=\left(\begin{array}{ccc}
1 & 0 & 0 \\
0 & -1 & 0 \\
0 & 0 & 0
\end{array}\right), & \lambda_{4}=\left(\begin{array}{lll}
0 & 0 & 1 \\
0 & 0 & 0 \\
1 & 0 & 0
\end{array}\right), \\
\lambda_{5}=\left(\begin{array}{ccc}
0 & 0 & -i \\
0 & 0 & 0 \\
i & 0 & 0
\end{array}\right), & \lambda_{6}=\left(\begin{array}{lll}
0 & 0 & 0 \\
0 & 0 & 1 \\
0 & 1 & 0
\end{array}\right),
\end{array}
$$


so that the measurement of $S_{x}$ assumes that the noncommuting observables $\lambda_{1}$ and $\lambda_{6}\left(\left[\lambda_{1}, \lambda_{6}\right]=\lambda_{4}\right)$ are measured simultaneously. At the same time, quantum uncertainty of the measurement of $S_{x}$ is higher than the common uncertainty of measurements of $\lambda_{1}$ and $\lambda_{6}$ :

$$
V\left(S_{x}\right)=\frac{1}{2}\left(V\left(\lambda_{1}\right)+V\left(\lambda_{2}\right)\right)+\operatorname{Cov}\left(\lambda_{1}, \lambda_{2}\right) .
$$

Here,

$$
V(X) \equiv\left\langle(X-\langle X\rangle)^{2}\right\rangle
$$

denotes the variance (uncertainty), and the covariance

$$
\operatorname{Cov}(X, Y)=\frac{1}{2}\langle X Y+Y X\rangle-\langle X\rangle\langle Y\rangle
$$

describes the quantum correlation between the measurements of two observables. Thus, reduction of the symmetry $\mathrm{SU}(3) \longrightarrow \mathrm{SU}(2)$ with the corresponding change of basic observables leads to an increase of uncertainty in measurements.

Hereafter we call the ternary quantum system with the SU(3) symmetry and observables (2) the true qutrit, and the system with the reduced symmetry SU(2) the $\mathrm{SU}(2)$ or spin qutrit.

In physics, the true qutrit is usually associated with the "eightfold way" and quark theory [11]. Nevertheless, some simple objects can also be associated with true qutrits. An example is provided by three-level atoms. In turn, the spin-qutrit represents a "spin-1" object that can be associated with spin, isotop-spin, angular momentum, etc. [12]. In the context of quantum information, the true qutrit can be realized as a quantum-dot cellular automata with eight quantum dots [13]. The simplest example of a spin-qutrit is provided by a biphoton $[14,15]$ (a couple of photons created simultaneously and propagating in the same direction).

Our analysis is based on the following key results.

(1) States carrying complete entanglement manifest maximal amount of total variance

$$
\mathbb{V}\left(\psi_{\mathrm{CE}}\right)=\max _{\psi \in \mathscr{H}} \mathbb{V}(\psi)
$$

Here,

$$
\mathbb{V}(\psi) \equiv \sum_{i} V\left(X_{i}\right)
$$

and summation is performed over all basic observables $X_{i}$ and over all parties in the case of multipartite system.

This condition (4) reflects the physical nature of quantum entanglement as a manifestation of quantum fluctuations at their extreme $[9,10,16]$.

(2) All entangled states of a given system are equivalent to within SLOCC (stochastic local operations assisted by classical communications). This property has been established in [17]. It has been shown in [18] that
SLOCC are represented by operators $g^{c}$ from the complexified dynamic symmetry group $G^{c}=\exp (\mathscr{L} \otimes \mathbb{C})$.

It follows from these two statements that all entangled states of a given system can be constructed from a certain generic CE state by means of SLOCC. A simple algorithm for construction of generic entangled states has been proposed in [19].

Entanglement of pure states of two true qutrits was discussed in [7] and within the frameworks of the dynamic symmetry approach in $[10,12,20]$. Below, we briefly discuss some important results.

The system is defined in the Hilbert space

$$
\mathscr{H}=\mathscr{H}_{3} \otimes \mathscr{H}_{3}
$$

and has the dynamic symmetry $\mathrm{SU}(3) \times \mathrm{SU}(3)$. The generic CE state of the system has the form $[1,19]$

$$
\begin{gathered}
\left|\psi_{\mathrm{CE}}\right\rangle=\frac{1}{\sqrt{3}}\left(|+1,+1\rangle+e^{i 2 k \pi / 3}|0,0\rangle+e^{i 4 k \pi / 3}|-1,-1\rangle\right), \\
k=0,1,2,
\end{gathered}
$$

where $|s, s\rangle \equiv|s\rangle \otimes|s\rangle$. This state can be considered as a generic entangled state of two true qutrits. This means that all other entangled states of this system can be defined as follows:

$$
\left|\psi_{E}\right\rangle=g_{\text {loc }}^{c}\left|\psi_{\mathrm{CE}}\right\rangle, \quad g_{\text {loc }}^{c}=\exp \left(z_{j} \lambda_{j}\right)
$$

where $z_{j}$ is a complex parameter. The amount of entanglement carried by two true qutrits can be measured by means of concurrence, which can be expressed in terms of the total variance as follows [21]:

$$
C(\psi)=\sqrt{\frac{\mathbb{V}(\psi)-\mathbb{V}_{\text {min }}}{\mathbb{V}_{\text {max }}-\mathbb{V}_{\text {min }}} .}
$$

It is seen that the CE state (6) provides the maximum total variance $\mathbb{V}_{\max }=32 / 3$. The minimal total variance $\mathbb{V}_{\text {min }}=8$ is given by the separable states that do not manifest entanglement. The generic state for this second class of states can be chosen as $| \pm 1, \pm 1\rangle$.

Unlike the case of two true qutrits, the states of two SU(2) qutrits can be divided into three classes. The class of entangled states is again generated from the state (6) through the use of SLOCC of the form

$$
g_{\text {loc }}^{c}=\exp \left(\xi_{i} S_{i}\right), \quad \xi_{i} \in \mathbb{C}, \quad i=x, y, z
$$

with the observables (3) instead of Gell-Mann matrices (2). The maximal total variance $\mathbb{V}_{\max }=2$ in this case. The second class is generated by SLOCC (9) from the state $|0,0\rangle$. In spite of its separability, this state also manifests the maximum of the total variance. Nevertheless, there is no contradiction with the above statement 1 . The point is that each party in $|0,0\rangle$ carries complete entanglement and that the total variance is additive with respect to contributions coming from the local parties.

Complete entanglement of a single SU(2) qubit in the state $|0\rangle$ has been shown in [20]. This fact follows 
Table

\begin{tabular}{l|l|l|l|l}
\hline \multicolumn{1}{c|}{ Type of state } & \multicolumn{1}{c|}{ Generic state } & Total variance & $\begin{array}{c}\text { Correlation } \\
\text { between parties }\end{array}$ & Correlation \\
\hline Entangled & Given by Eq. (7) & $\mathbb{V}_{\max } \geq \mathbb{V}>\mathbb{V}_{\text {min }}$ & Strong correlation & No correlations \\
Separable entangled & $|0,0\rangle$ & $\mathbb{V}_{\max } \geq \mathbb{V}>\mathbb{V}_{\min }$ & No correlations & Strong correlation \\
Unentangled & $| \pm 1, \pm 1\rangle$ & $\mathbb{V}=\mathbb{V}_{\min }$ & No correlations & No correlations \\
\hline
\end{tabular}

from the equivalence of two qubits with basic states, forming a symmetric triplet, and single SU(2) qutrit. As an illustrative example of some considerable interest, the states of biphoton $[14,15]$ can be mentioned. In terms of the horizontal $(H)$ and vertical $(V)$ polarizations forming a qubit for each photon, the basic states are denned as follows:

$$
|V, V\rangle, \quad \frac{1}{\sqrt{2}}(|V, H\rangle+|H, V\rangle), \quad|H, H\rangle .
$$

The antisymmetric polarization state of a biphoton is forbidden due to the Bosonic nature of this object. It is clear that the states with parallel polarizations $|V, V\rangle$ and $|H, H\rangle$ can be associated with the spin-projection states $|1\rangle$ and $|-1\rangle$ in (1). In turn, the state with indefinite polarization in (10) corresponds to the spin-projection state $|0\rangle$ in (1). This state is certainly completely entangled with respect to polarization of photons. In more general settings, a single $S U(2)$ qutrit in the state $|0\rangle$ is completely entangled with respect to certain intrinsic degrees of freedom. For physical examples, see [12, 20].

To be more rigorous, we note that existence of the single SU(2)-qutrit entanglement follows directly from the Clebsch-Gordon decomposition of the Hilbert space of two qubits:

$$
\mathscr{H}_{2} \otimes \mathscr{H}_{2}=\mathscr{H}_{3} \oplus \mathscr{H}_{A},
$$

where $\mathscr{H}_{2}$ denotes the single-qubit space of states and $\mathscr{H}_{A}$ corresponds to the antisymmetric singlet state.

The principle difference between the complete entangled state (6) and separable entangled state $|0,0\rangle$ can also be illustrated by the fact that local measurements between the two parties are strongly correlated in the former case and completely uncorrelated in the latter case. In other words, both states carry complete entanglement. In state (6), it is caused by the quantum correlations between parties of the system, which corresponds to the traditional interpretation of entanglement. In the state $|0,0\rangle$, entanglement has a completely local nature. The intrinsic degrees of freedom are responsible for this kind of entanglement.

Besides that, it is easily seen that the states (6) and $|0,0\rangle$ are nonequivalent with respect to SLOCC (9).

The third class of states is formed by unentangled states that can be generated from $| \pm 1, \pm 1\rangle$ by means of SLOCC (9). They are separable as well as $|0,0\rangle$ and do not manifest quantum correlation between parties either. Nevertheless, they are completely different because of the principle difference between the single SU(2) qutrit states $|0\rangle$ and $| \pm 1\rangle$. As we have seen, the former one is completely entangled. The latter states can be associated with the $\mathrm{SU}(2)$ coherent states that can be defined as follows [22]:

$$
\begin{gathered}
|\alpha\rangle=\exp \left(\alpha S_{+}-\alpha^{*} S_{-}\right)|-1\rangle \\
=\frac{e^{2 i \phi}}{2}(1-\cos 2|\alpha|)|1\rangle+\frac{i e^{i \phi}}{\sqrt{2}} \sin 2|\alpha||0\rangle \\
+\frac{1}{2}(1+\cos 2|\alpha|)|-1\rangle,
\end{gathered}
$$

where $S_{ \pm}=S_{x} \pm i S_{y}, \alpha$ is a complex parameter, and $\phi=$ $\arg \alpha$. At $|\alpha|=(2 k+1) \pi / 2$, this state coincides with $|1\rangle$, at $|\alpha|=k \pi$, with $|-1\rangle$. It can never coincide with $|0\rangle$, which is the spin-squeezed rather than spin-coherent state [12].

The properties of states of two $\mathrm{SU}(2)$ qutrits are listed in the following table.

Concurrence for the class of entangled states is given by Eq. (8). If we choose a general state of a single $\mathrm{SU}(2)$ qutrit as

$$
|\psi\rangle=a|1\rangle+b|0\rangle+c|-1\rangle, \quad|a|^{2}+|b|^{2}+|c|^{2}=1,
$$

the single-party entanglement for the separable entangled states is specified by the concurrence [20]

$$
C(\psi)=2\left|a c-b^{2} / 2\right| .
$$

Summarizing, we have considered the principle difference between entanglement of pure states of two true qutrits and two SU(2) qutrits caused by the change of the amount of quantum information due to the lack of observables and increase of uncertainty in the latter case. The space of states of two SU(2) qutrits has an additional class of separable entangled states that carry entanglement of intrinsic degrees of freedom in each party while having no quantum correlations between parties. This example is of special interest. In a sense, a system of two SU(2) qutrits is equivalent to the system of four qubits defined in the symmetric subspace of $\mathscr{H}=\mathscr{H}_{2} \otimes \mathscr{H}_{2} \otimes \mathscr{H}_{2} \otimes \mathscr{H}_{2}$, so that the above-mentioned class of states exists in this case as well.

Note that existence of entanglement for a part of a multipartite system can also be observed for three qubits. Namely, the so-called $W$ state does not manifest 
entanglement in the three-partite sector, while is entangled in the bipartite sector [23].

The classes of entangled states of two true and SU(2) qutrits have the same generic state (6). This means that entanglement of true qutrits involves entanglement of the SU(2) qutrits, but not vice versa.

As a hint for practical distinguishability among the entangled and separable entangled states of two SU(2) qutrits, we note that both parties in the former class manifest the same amount of total variance, while the amount of the single-party variance is different for separable entangled states (except the generic state $|0,0\rangle$ ).

Above, we have considered pure states. Classification of mixed states of qutrits deserves special consideration.

\section{ACKNOWLEDGMENTS}

One of the authors (B.Ö.) would like to acknowledge the scientific and Technical Research Council of Turkey (TÜBİTAK) for financial support.

\section{REFERENCES}

1. A. V. Burlakov, M. V. Chechova, O. A. Karabutova, et al., Phys. Rev. A 60, R4209 (1999).

2. H. Bechman-Pasquinucci and A. Peres, Phys. Rev. Lett. 85, 3313 (2000).

3. D. Bruß and C. Macchiavello, Phys. Rev. Lett. 88, 127901 (2002).

4. G. A. Maslennikov, A. A. Zhukov, M. V. Chechova, and S.P. Kulik, J. Opt. B: Quantum Semiclass. Opt. 5, 530 (2003).

5. D. Kaszlikowski, D. K. L. Oi, M. Christandl, et al., Phys. Rev. A 67, 012310 (2003).
6. T. Durt, N. Cerf, N. Gisin, and M. Zukowski, Phys. Rev. A 67, 012311 (2003).

7. C. M. Caves and G. J. Milburn, Opt. Commun. 179, 439 (2000).

8. R. Hermann, Lie Groups for Physicists (Benjamin, New York, 1966).

9. A. A. Klyachko, quant-ph/0206012.

10. A. A. Klyachko and A. S. Shumovsky, J. Phys.: Conf. Ser. 36, 87 (2006).

11. A. Watson, The Quantum Quark (Cambridge Univ. Press, New York, 2004).

12. S. Binicioğlu, M. A. Can, A. A. Klyachko, and A. S. Shumovsky, quant-ph/0604182.

13. I. L. Bajec, N. Zimic, and M. Mraz, Nanotechnology 17, 1937 (2006).

14. M. V. Chechova, L. A. Krivitsky, S. P. Kulik, and G. A. Maslennikov, Phys. Rev. A 70, 053801 (2004).

15. Yu. I. Bogdanov, M. V. Chekhova, S. P. Kulik, et al., Phys. Rev. Lett. 93, 230503 (2004).

16. A. A. Klyachko and A. S. Shumovsky, J. Opt. B: Quantum Semiclass. Opt. 6, S29 (2004).

17. W. Dür, G. Vidal, and J. I. Cirac, Phys. Rev. A 62, 062314 (2000).

18. F. Verstraete, J. Dehaene, and B. De Moor, Phys. Rev. A 68, 012103 (2003).

19. S. Binicioğlu, Ö. Çakir, A. A. Klyachko, and A. S. Shumovsky, Int. J. Quantum Inform. 3, 661 (2005).

20. M. A. Can, A. A. Klyachko, and A. S. Shumovsky, J. Opt. B: Quantum Semiclass. Opt. 7, L1 (2005).

21. A. A. Klyachko, B. Öztop, and A. S. Shumovsky, Appl. Phys. Lett. 88, 124102 (2006).

22. J. M. Radcliffe, J. Phys. A 4, 313 (1971); F. T. Arecchi, E. Courtens, R. Gilmore, and H. Thomas, Phys. Rev. A 6, 2211 (1972).

23. A. Miyake, Phys. Rev. A 67, 012108 (2003). 